\title{
Solvothermal Clustering of Magnetic Spinel Ferrite Nanocrystals: A Raman Perspective
}

\author{
Ruth Otero-Lorenzo, ${ }^{\dagger}$ Miguel A. Ramos-Docampo, ${ }^{\dagger}$ Benito Rodríguez-González, ${ }^{\dagger \S}$ Miguel Come- \\ saña-Hermo, ${ }^{+*}$ Verónica Salgueiriño ${ }^{\dagger}$
}

† Departamento de Física Aplicada, Universidade de Vigo, 36310 Vigo, Spain

$\S$ CACTI, Universidade de Vigo, 36310 Vigo, Spain

\begin{abstract}
The one-pot formation of colloidal nanocrystal clusters has recently gained attention due to the new set of phenomena that such secondary structures are endowed with. Nevertheless, given the number of complex processes at play, the correct interpretation of the chemical mechanisms behind the formation of such structures remains a particularly difficult task. In the present work, magnetic colloidal nanocrystal clusters with a spinel structure are thoroughly characterized by means of transmission electron microscopy, X-ray diffraction, magnetic measurements and Raman spectroscopy. The combination of these techniques results in a means to unravel the structure of the poorly crystalline intermediates and complex products formed throughout the process. Our results suggest that the chemical and crystallographic transformations undergone by the nanostructures at the different stages of the reaction are strongly related with the final morphology of the secondary super-structures obtained, which in parallel, help to improve the magnetic properties required in their specific final applications.
\end{abstract}

\section{INTRODUCTION}

The development of the colloidal synthesis of nanoparticles has led to an impressive portfolio of nanomaterials with different sizes, compositions and morphologies that are currently available. ${ }^{1-3}$ As the synthesis of individual nanocrystals has been gradually mastered, the attention of the scientific community has recently shifted towards the use of these well-studied nanoparticles as building-blocks for the formation of more complex materials. Colloidal nanocrystal clusters (CNCs) are a very interesting example of this kind of superstructures. The physical and chemical interactions established between their individual units lead to a unique set of physical phenomena that can be used for the formation of plasmonic metamaterials, ${ }^{4}$ the creation of hot-spots for surface enhanced Raman spectroscopy (SERS), ${ }^{5}$ novel magnetoelectric multiferroic materials ${ }^{6}$ or diagnostic and therapeutic agents with improved magnetic performance for magnetic guidance and separation or heat delivery applications, ${ }^{7}$ among a vast set of potential applications.

CNCs are the result of the interplay between different forces such as entropy-driven, electrostatics, van der Waals interactions or hydrogen bonding. ${ }^{8}$ The control of these interactions together with conventional reaction parameters (surfactants, temperature, reaction time or solvent used) can lead to the successful control over the morphology and composition of both the primary crystallites and the CNCs. ${ }^{9}$ It is well established that the synthesis of these structures is accomplished following one of the following pathways: (i) direct synthesis of CNCs in solu- tion or (ii) a consecutive multi-step process in which pre-synthesized nanocrystals become aggregated in a controlled manner thanks to the use of external stimuli such as temperature, $\mathrm{pH}$, light or redox potential. ${ }^{10}$ In the latter case, the use of multiple steps implies tedious and repetitive purifications and, in most cases, changes in the surface functionalities of the nanoparticles in order to favor the controlled aggregation. ${ }^{11}$ Along these lines, the one-pot synthesis is a simpler, greener and more cost-efficient alternative for the formation of CNCs.

Among the plethora of inorganic CNCs available, those formed by the controlled aggregation of magnetic units are particularly relevant given the collective magnetic phenomena that arise in such hierarchical structures, ${ }^{9}$ with important consequences in their performance for multiple applications. In this vein, dipolar interactions between magnetic units play a prominent role over the final magnetic response of the ensemble: while weak interparticle forces may lead to superparamagnetic $\mathrm{CNCs}$, a stronger coupling can result in a net coherent magnetization and thus, the formation of a ferromagnetic structure at room temperature. The compromise between these two situations renders the CNCs very attractive as suitable for both magnetic guidance without aggregation in the first case or for heat delivery in the second one. Indeed, concerning the magnetic performance, there are two key actions these CNCs can accomplish better than single nanoparticles, given their unique morphology and the consequent dipolar interactions established in it. For example, Rikken et al. have revised the manipulation of micro- and 

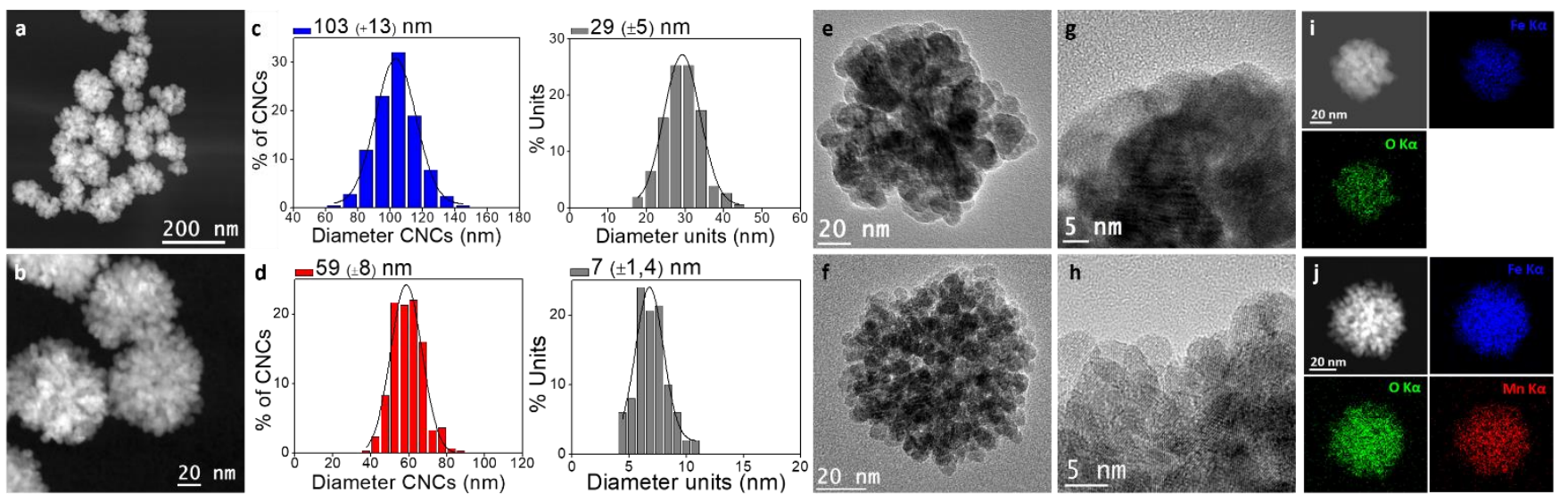

Figure 1. TEM characterization of the $\mathrm{Fe}_{3} \mathrm{O}_{4}$ (upper panel) and $\mathrm{Mn}_{0.6} \mathrm{Fe}_{2.4} \mathrm{O}_{4}$ (lower panel) CNCs. (a, b) STEM-HAADF images, (c, d) size histograms, (e, f) TEM images, (g, h) HRTEM images and (i, j) EDS elemental mappings from each sample.

nanostructures motion with magnetic fields. ${ }^{12}$ This is indeed very useful for example in the case of self-propelled superparamagnetic superstructures, which become accelerated due to the exerted magnetic force and given the much larger total magnetic moment of these structures. ${ }^{13}$ Alternatively, Respaud and coworkers have elucidated via kinetic Monte Carlo simulations the way to increase the magnetic hyperthermia properties of this kind of superstructures attending to key parameters such as magnetocrystalline anisotropy, while controlling the dipolar interactions. $^{14}$

Even though an important number of publications deal with the one-step synthesis and characterization of magnetic CNCs, little experimental evidence of the chemical mechanisms behind the formation of such superstructures can be found in the literature. This lack of information stems from the unstable intermediates formed throughout the synthetic procedure and the advanced and complex techniques needed to characterize them. Therefore, a multidisciplinary approach with the right combination of different tools to study the intermediate species formed needs to be implemented. Along these lines, a recent work by Cannas and coworkers presents a comprehensive study in which the synthetic mechanism leading to the one-pot formation of $\mathrm{CoFe}_{2} \mathrm{O}_{4} \mathrm{CNCs}$ in aqueous solution is investigated in detail. Nevertheless, this work is focused in the morphological changes undergone by the crystallites (analyzed by transmission electron microscopy (TEM), X-ray diffraction (XRD) and small angle X-ray scattering (SAXS)) rather than in the chemistry of the intermediates. ${ }^{15}$

Consequently, the objective of the present work is to shade light over the chemical mechanism that governs the formation of a particular type of secondary superstructures. In order to do this, we have studied the temporal evolution of the reactions involved, using different but complementary techniques to characterize in detail every step of the process. Among them, Raman spectroscopy has proven to be a particularly valuable tool to obtain further insight into the chemical composition of the intermediates formed, allowing us to postulate a mechanism responsible for the final morphology and composition and consequently the magnetic properties of the CNCs obtained.

EXPERIMENTAL SECTION
Reagents. Iron (III) chloride hexahydrate (>99\%), manganese (II) chloride tetrahydrate $(>98 \%)$, sodium acetate $(>99 \%)$, poly(ethylene glycol) (MW 6,000) and ethylene glycol (>99\%) were purchased from Sigma-Aldrich. All chemicals were used as received. Milli-Q grade water was used in all preparations.

Instrumentation. Transmission electron microscopy measurements were performed on a JEOL JEM 1010 instrument operating at an acceleration voltage of $100 \mathrm{kV}$. High resolution (HR)-TEM analysis and energy-dispersive X-ray spectroscopy (EDS) were carried out on a field emission JEOL JEM2010F instrument working at $200 \mathrm{kV}$. Samples for the TEM analysis were prepared by dropping a diluted suspension of the CNCs onto an ultrathin carbon-coated copper grid. X-ray diffraction (XRD) patterns were collected by using a Siemens D-5000 powder X-ray diffractometer (CuKa radiation (l=1.54056 $\AA$ ) in the range $2 \theta=5-100^{\circ}$ ) and compared with the crystallographic information files (CIF) from the crystallographic open database (COD). Raman spectra were collected in powder samples onto a glass slide as substrate, with a Renishaw in Via Reflex Raman Microscope. Experiments were conducted at room temperature by using an excitation wavelength of $785 \mathrm{~nm}$ (near-IR diode). Magnetic measurements were performed by using a Quantum Design MPMS XL7 magnetometer.

Synthetic procedure. The synthesis of $\mathrm{Fe}_{3} \mathrm{O}_{4}$ and $\mathrm{Mn}_{\mathrm{x}} \mathrm{Fe}_{3-\mathrm{x}} \mathrm{O}_{4}$ $\mathrm{CNCs}$ proceeds through the modification of a previously published procedure by Deng and coworkers. ${ }^{16}$ It consists in the partial reduction of hexahydrated $\mathrm{FeCl}_{3}$ and tetrahydrated $\mathrm{MnCl}_{2}$ (the different $\mathrm{Mn}^{2+} / \mathrm{Fe}^{3+}$ ratio permits tuning the stoichiometric $x$ value) in the presence of polyethylene glycol (PEG) and sodium acetate (NaAc), using ethylene glycol (EG) as both solvent and reducing agent. The mixture formed is homogenized and finally introduced into a stainless steel picoclave $(100$ $\mathrm{mL}$ ), where the solvothermal process takes place at $185{ }^{\circ} \mathrm{C}$. Once the reaction proceeds for the desired amount of time, the solution is cooled to room temperature and washed several times to remove excess reactants. The solid obtained is dried overnight at $60{ }^{\circ} \mathrm{C}$ in an oven for further characterizations. Herein, the reaction has been stopped at four different reaction times (30 minutes, 1 hour, 3 hours and 8 hours) in order to characterize all the intermediate products obtained at the different 

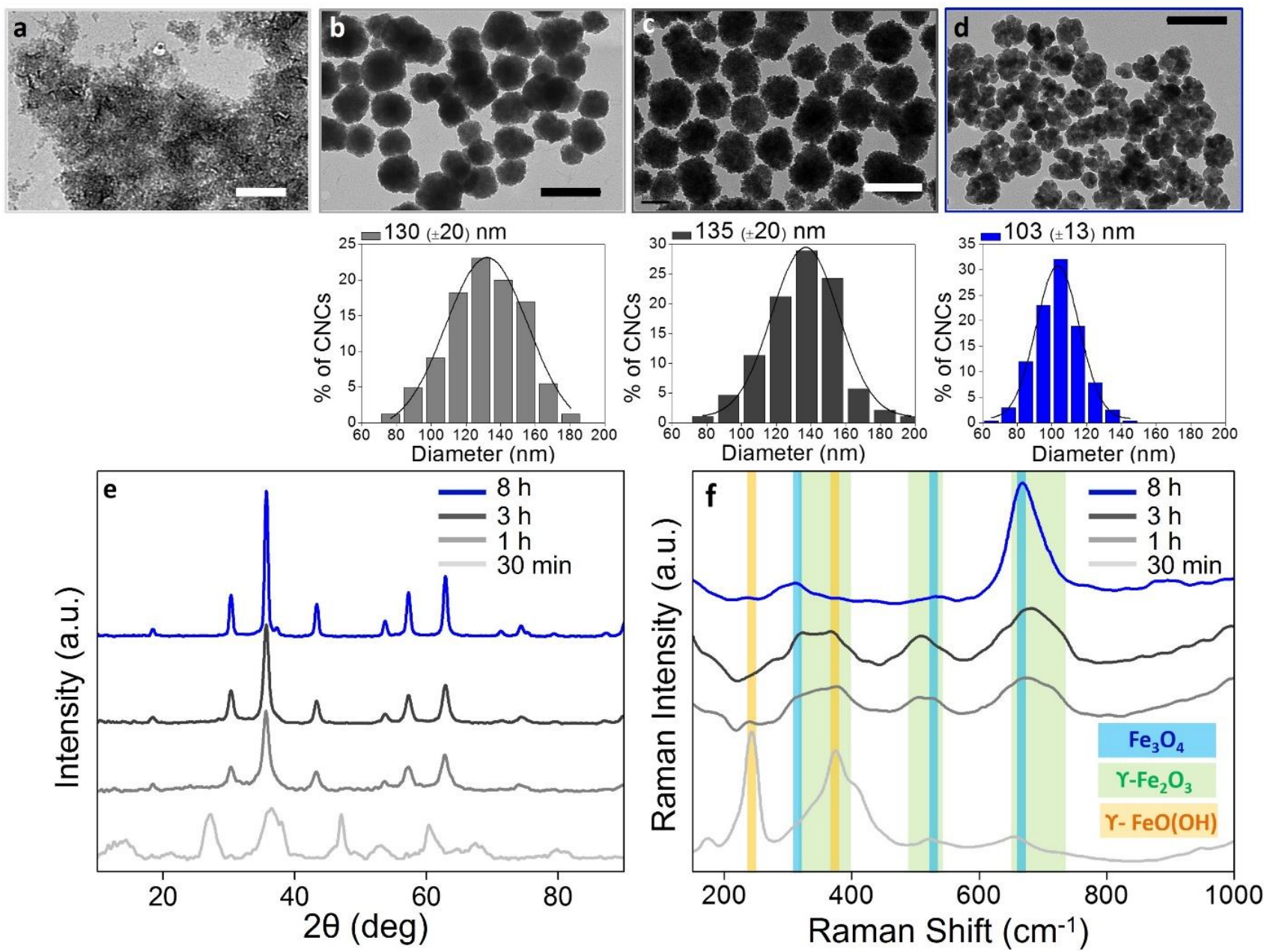

Figure 2. (a-d) TEM images of the products obtained in the synthesis of $\mathrm{Fe}_{3} \mathrm{O}_{4}$ CNCs at different reaction times: (a) 30 minutes, (b) 1 hour, (c) 3 hours and (d) 8 hours (scale bar: $200 \mathrm{~nm}$ ) with their respective size histograms. (e) X-ray diffraction patterns of the same samples and (f) their corresponding Raman spectra $\left(\lambda_{\text {exc }}: 785 \mathrm{~nm}\right)$.

stages of the reaction and understand the role that they may play in the growth mechanism of the CNCs.

\section{RESULTS AND DISCUSSION}

Figure 1 includes the complete TEM analysis of $\mathrm{Fe}_{3} \mathrm{O}_{4}$ and $\mathrm{Mn}_{\mathrm{x}} \mathrm{Fe}_{(3-\mathrm{x})} \mathrm{O}_{4} \mathrm{CNCs}$ obtained after 8 hours of reaction. The images presented herein show that the mean diameter of the magnetite CNCs is larger $(103 \pm 13 \mathrm{~nm})$ than that observed when $\mathrm{Mn}$ is introduced into the spinel structure $(59 \pm 8 \mathrm{~nm})$. Moreover, the size of the individual units forming the clusters differ from one sample to another, being again bigger those of the magnetite sample ( $29 \mathrm{~nm}$ vs. $7 \mathrm{~nm}$ ). Interestingly, HRTEM characterization shows the crystalline nature of those individual units forming the CNCs after 8 hours of solvothermal treatment (Figure 1g, h). EDS elemental mappings show that the distributions of $\mathrm{Fe}$ and $\mathrm{O}$ are homogeneous throughout the CNCs (Figure 1i). The same is observed for the $\mathrm{Mn}_{\mathrm{x}} \mathrm{Fe}_{(3-\mathrm{x})} \mathrm{O}_{4} \mathrm{CNCs}$, with homogeneous distributions of $\mathrm{Mn}, \mathrm{Fe}$ and $\mathrm{O}$ (Figure 1j). Moreover, quantitative analysis of the chemical composition of the Mn-doped sample leads to a chemical composition that corresponds to the stoichiometric ratio $\mathrm{Mn}_{0.6} \mathrm{Fe}_{2.4} \mathrm{O}_{4}$.

As mentioned above, a thorough characterization of the intermediates formed in these processes has been performed. In order to do this, a complete characterization of the products obtained at four different reaction times (30 minutes and 1, 3 and 8 hours) has been undertaken. For the sake of clarity, the results obtained for $\mathrm{Fe}_{3} \mathrm{O}_{4}$ and $\mathrm{Mn}_{0.6} \mathrm{Fe}_{2.4} \mathrm{O}_{4}$ CNCs are presented in a sequential fashion.

TEM investigations of the products obtained for the synthesis of magnetite $\mathrm{CNCs}$ at different reaction times have been performed in order to study the time evolution of their morphology (Figure 2a-d for the $\mathrm{Fe}_{3} \mathrm{O}_{4}$ case). After $30 \mathrm{~min}$ of reaction only few elongated nanostructures trapped in an amorphous matrix (without formation of well-defined nanoparticles or clusters) can be observed (Figure 2a and S1), in agreement with the direct synthesis of CNCs in solution: the primary nanoparticles are formed in a super-saturated solution prior to their subsequent organization into clusters. After longer periods of time (1 and 3 h) the colloidal clusters of nanoparticles are already formed (Figure 2b, c). Surprisingly, the sizes are larger in both cases $(131 \pm 21 \mathrm{~nm}($ after $1 \mathrm{~h})$ and $135 \pm 21 \mathrm{~nm}($ after $3 \mathrm{~h}))$, than those obtained at the end of the reaction, i.e. $8 \mathrm{~h}(103 \pm 13 \mathrm{~nm})$ (Figure $2 d)$. On the other hand, the size of the nanocrystals that form the aggregates at this stage $(3 \mathrm{~h})$ are rather small $(11 \pm 1 \mathrm{~nm})$ with respect to the final product $(29 \pm 5 \mathrm{~nm}$ ) (Figure 1c and SI1). Generally, this type of processes can be related to an Ostwald ripening, in this case an intra-cluster Ostwald ripening process that takes place between crystallites present in the same super-structure, thus allowing the larger nuclei to grow at the expense of the smaller ones. Following this assumption, the reduction in the final diameter of the clusters may be the direct consequence of a decrease in their interstitial free space after 
rearrangement of the larger crystallites into a thermodynamically more stable disposition. ${ }^{17}$

Examination of the magnetite powders obtained with X-ray diffraction shows two different patterns (Figure 2e). Firstly, the reddish powder obtained after 30 minutes (without response when an external magnetic field is applied) presents wide diffraction peaks that can be assigned to lepidocrocite $(\gamma-$ $\mathrm{FeO}(\mathrm{OH})$ ), an iron oxyhydroxide mineral that presents an orthorhombic crystalline structure. The broad shape of the peaks is the consequence of the small size of the crystalline domains obtained in the early stages of the reaction. It is important to highlight that the reaction mixture only reaches the final temperature $\left(185^{\circ} \mathrm{C}\right)$ inside the reactor 45 minutes after the reaction has started. Accordingly, the limited reducing power of EG at short reaction times should have a strong impact in the oxidation state and crystallinity of the species obtained at short reaction times. On the other hand, the powders obtained for the three longer reaction times (1, 3 and 8 hours) show very similar profiles that can be assigned to either magnetite, maghemite or a mixture of them since both iron oxides present the same spinel structure and thus, their XRD patterns are extremely difficult to discriminate from one another. Even though these three spectra show the same diffraction peaks, the full widths at half maximum (FWHM) are reduced with reaction time. This behavior is a direct consequence of the increase in the size of the crystalline domains that form the CNCs (as previously observed by TEM) and confirmed by the size of the crystalline domains obtained from the XRD data using the Debye-Scherrer formula (Table S1).

In view of the limited information provided by X-ray diffraction we complete the structural analysis using Raman spectroscopy. This technique is a powerful means to investigate structural properties of materials, and can provide unique information when analyzing nanostructured transition metal oxides. ${ }^{18-21}$ It is indeed a very convenient technique, since it can register the different vibrations due to the different cationic arrangement in the magnetite or mixed manganese ferrite-based NPs presented here. ${ }^{7}$ Since the materials object of this study are particularly prone to oxidation, we have used a laser with an excitation wavelength of $785 \mathrm{~nm}$ (but with very low power) to achieve a satisfactory penetration depth in the sample and avoid therefore oxidation artifacts that could emerge when measuring superficial areas.

The Raman spectrum of the magnetite sample obtained after 30 minutes of reaction shows two main bands at 243 and $374 \mathrm{~cm}^{-1}$ accompanied by less intense peaks at 519 and $651 \mathrm{~cm}^{-1}$ and a shoulder at $406 \mathrm{~cm}^{-1}$ (Figure 2f). While the four first peaks can be undoubtedly assigned to lepidocrocite (the colored shadows in Figure $2 \mathrm{f}$ serve as a guide to the eye), the latter could appear due to the presence in the sample of concomitant feroxyhyte $(\delta$ $\mathrm{FeO}(\mathrm{OH}))^{22}$ This result points out that the experiment indeed goes first through the lepidocrocite phase, whose formation may be the result of the hydrolysis of ferric chloride under the basic conditions provided by sodium acetate. In these early stages of the reaction the reducing power of EG is limited since the conditions of temperature and pressure needed have not been met yet, allowing therefore the formation of such metastable intermediate. Attending to the subsequent Raman spectrum obtained after $1 \mathrm{~h}$, it is clear that the peaks of lepidocrocite start to vanish while broad bands appear at 340,510 and $675 \mathrm{~cm}^{-1}$, corresponding to the $\mathrm{T}_{2 \mathrm{~g}}, \mathrm{E}_{\mathrm{g}}$ and $\mathrm{A}_{1 \mathrm{~g}}$ modes of maghemite, respectively. ${ }^{22,23}$ Even though it could be discussed that the latter signal arises due to the presence of an important amount of magnetite that overlaps the phonon mode of maghemite, previous reports highlight the broad and asymmetric band of the maghemite $A_{1 g}$ mode (i.e. like the one obtained in this study), in opposition to a thin symmetric and intense peak in the case of magnetite. Therefore, this broad band centered at $675 \mathrm{~cm}^{-1}$ is mainly due to the presence of maghemite (even though a simultaneous amount of magnetite cannot be discarded). Accordingly, the first product obtained (lepidocrocite) experiences a phase transition as the temperature increases during the course of the reaction, leading to the formation of the more stable maghemite phase. The only significant difference between the spectrum obtained after $3 \mathrm{~h}$ and the previous one is the complete disappearance of the band at $243 \mathrm{~cm}^{-1}$ and therefore the complete vanishing of remnant lepidocrocite. Once the reaction is completed $(8 \mathrm{~h})$ we can only observe the features due to the presence of magnetite: $313 \mathrm{~cm}^{-1}\left(\mathrm{E}_{\mathrm{g}}\right), 530 \mathrm{~cm}^{-1}\left(\mathrm{~T}_{2 \mathrm{~g}}\right)$ and 671 $\mathrm{cm}^{-1}\left(\mathrm{~A}_{1 \mathrm{~g}}\right)$ that appear after reduction of maghemite due to the higher reducing power of PEG and EG. Other characteristic features of this material like the black coloration of its powders and their strong interaction with external magnetic fields were also observed in the sample isolated at the end of the process.

As previously mentioned, the same characterization tools have been used to unravel the mechanism governing the formation of the $\mathrm{Mn}_{0.6} \mathrm{Fe}_{2.4} \mathrm{O}_{4} \mathrm{CNCs}$. As we will see in the following, we have found important differences in this case with respect to the formation of the magnetite CNCs. TEM images of the product obtained after $30 \mathrm{~min}$ of reaction show the presence of an irregular solid (Figure 3a). From the contrast of the images we can argue that at this stage the sample may be composed by an amorphous inorganic solid in which the metallic ions $\left(\mathrm{Fe}^{3+}\right.$ and $\mathrm{Mn}^{2+}$ ) are embedded. After 1 hour, the morphology of the product has changed, showing elongated nanostructures with a mean length of ca. $35 \mathrm{~nm}$ (Figure 3b). In opposition to the synthesis of magnetite where small spherical nanoparticles were trapped into CNCs at this stage of the reaction, in the present case irregular aggregates composed by elongated objects are obtained, highlighting the important effect that $\mathrm{Mn}^{2+}$ ions exert in the kinetics of the reaction. Actually, it is only after 3 hours of reaction when CNCs are formed (Figure 3c). Moreover, the clusters obtained here at this intermediate stage are smaller $(61 \pm 7 \mathrm{~nm})$ than those formed for magnetite at the same reaction time (135 $\pm 21 \mathrm{~nm})$. Finally, once the reaction is finished $(8 \mathrm{~h})$ we observe that the morphology of the CNCs has not changed significantly since they present almost the same size $(59 \pm 8 \mathrm{~nm})$ than those obtained after $3 \mathrm{~h}$ (Figure 3d). Interestingly, the size of the NPs that form these clusters has experienced a limited increase in diameter with respect to those obtained at shorter reaction times (from $5.5 \pm 1.0 \mathrm{~nm}$, they reach a final average diameter of $7.0 \pm$ $1.4 \mathrm{~nm}$ ) (Figure S2), being also much smaller than those obtained for magnetite. This last result implies that a possible Ostwald ripening process has been slowed down by the presence of $\mathrm{Mn}$, demonstrating again the importance of this species in the evolution of the reaction.

The XRD analysis of these four samples show two main patterns (Figure 3e). For the shorter reaction times (30 min and 1 h) we obtain two very similar patterns with the same broad and weak diffraction peaks due to the poorly crystalline nature of the products at these stages of the reaction. The relatively good correspondence of these diffraction peaks with those obtained in the previous experiment after $30 \mathrm{~min}$ of reaction leads us to postulate again the formation of lepidocrocite. Nevertheless, the noisy patterns and small variations in the position of the peaks 

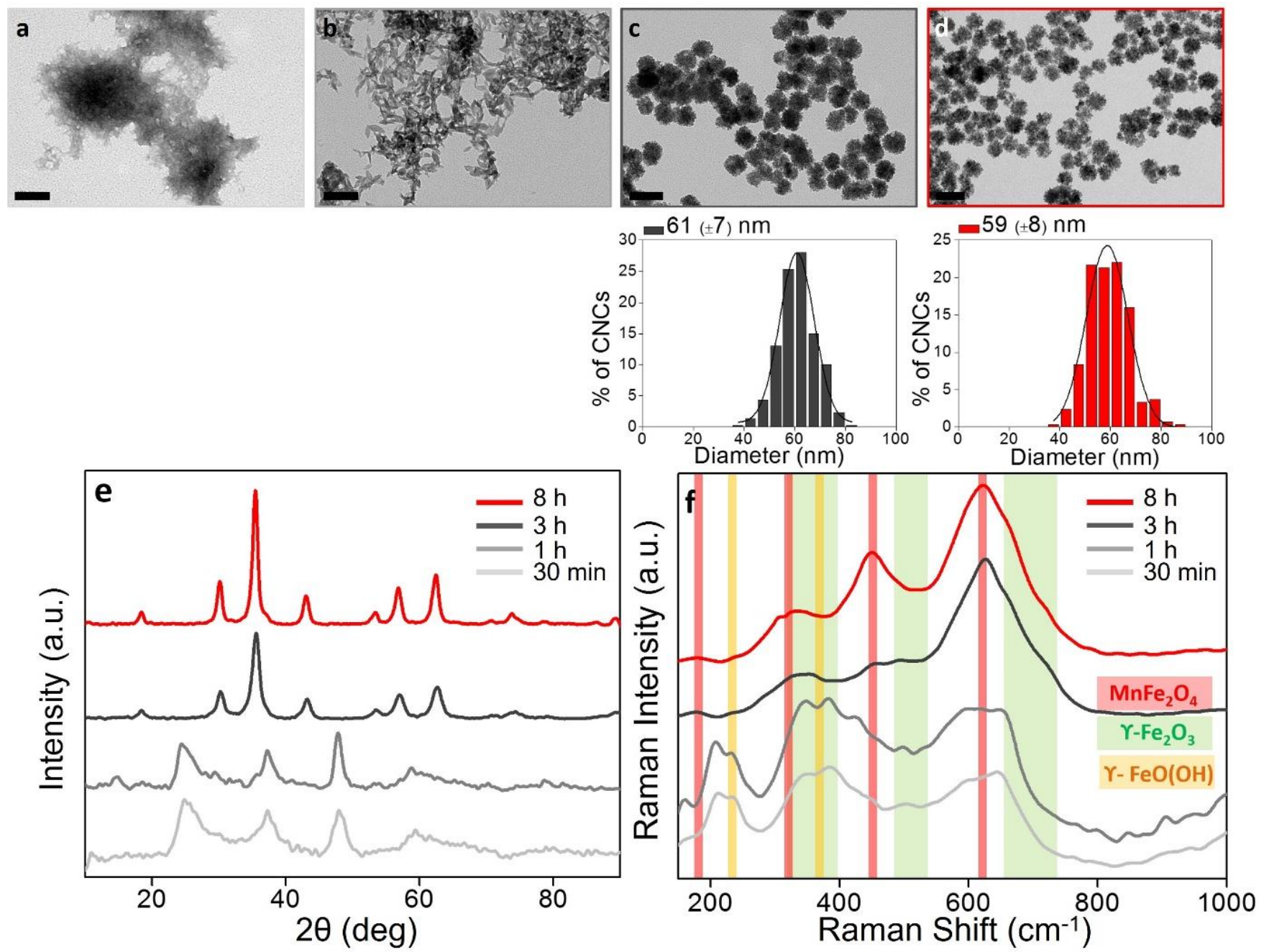

Figure 3. (a-d) TEM images of the products obtained in the synthesis of $\mathrm{Mn}_{0.6} \mathrm{Fe}_{2.4} \mathrm{O}_{4} \mathrm{CNCs}$ at different reaction times: (a) 30 minutes, (b) 1 hour, (c) 3 hours and (d) 8 hours (scale bar: $100 \mathrm{~nm}$ ) with their respective size histograms. (e) X-ray diffraction patterns of the same samples and (f) their corresponding Raman spectra $\left(\lambda_{\text {exc }}: 785 \mathrm{~nm}\right)$.

obtained with respect to reference values emphasize that XRD data alone is not sufficient to support this hypothesis (Figure S3). Therefore, the Raman spectroscopy results presented below are of valuable importance to confirm this assumption. A completely different scenario is observed for the XRD patterns obtained at longer reaction times ( 3 and $8 \mathrm{~h}$ ). In both cases we observe well-defined peaks that correspond to a spinel structure. As previously explained, XRD data of spinels (i.e. maghemite, magnetite or transition metal ferrites) show the same diffraction patterns since all of them share the same crystalline structure. Therefore, Raman spectroscopy will be used to extend the information given by TEM and X-ray diffraction data. The Raman spectra of the poorly crystalline solid obtained after $30 \mathrm{~min}$ and $1 \mathrm{~h}$ of reaction are shown in Figure 3f. In comparison with the spectrum obtained at the same reaction time but without presence of Mn, we can observe the appearance of more bands, leading to a more complex spectrum. Among them, we can observe peaks at 207 (with a shoulder at 231), 384 and 507 $\mathrm{cm}^{-1}$ together with a broad band centred at $633 \mathrm{~cm}^{-1}$. Even though there are significant shifts with respect to the peaks obtained in Figure 2f, we ascribe these four signals to the presence of lepidocrocite in the sample. Moreover, we have tried to assign the other peaks to $\mathrm{Mn}^{2+}$ and $\mathrm{Mn}^{3+}$ species that could be formed at the first stages of the reaction (i.e. $\mathrm{MnO}, \mathrm{Mn}(\mathrm{OH})_{2}$, $\mathrm{Mn}_{2} \mathrm{O}_{3}$ or $\left.\mathrm{MnO}(\mathrm{OH})\right)$. Nevertheless, none of these species could be fitted with our data. Therefore, the new signals together with the small divergences present in the previous peaks are a consequence of the doping of the solid with interstitial $\mathrm{Mn}^{2+}$ cations. The presence of this species leads to a loss in symmetry of the crystalline matrix (i.e. greater disorder) and the subsequent appearance of new active Raman modes (the presence of phonons is specific for each sample and consequently, non-stoichiometry, presence of vacancies, interstitial cations, and defects in general can result in the activation of new phonon modes that are not predicted by group theory). ${ }^{24}$ This effect can be also responsible for the small variations between the positions of the peaks in the XRD patterns obtained for magnetite and mixed manganese iron ferrite at 30 minutes of reaction (Figure SI2).

The spectra of the same product after 3 and 8 hours show four main features: a very broad $\left(550-700 \mathrm{~cm}^{-1}\right)$ band centred at 620 $\mathrm{cm}^{-1}$ and three weaker features at 448,330 and $180 \mathrm{~cm}^{-1}$. All these peaks can be directly related to the manganese ferrite pattern $\left(\mathrm{A}_{1 \mathrm{~g}}, \mathrm{~T}_{2 \mathrm{~g}(2)}, \mathrm{E}_{\mathrm{g}}\right.$ and $\mathrm{T}_{2 \mathrm{~g}(1)}$, respectively) as compared with the spectrum reported by Pereira and co-workers (showed as red areas in Figure 3f) while the missing $\mathrm{T}_{2 \mathrm{~g}(3)}$ mode may be shielded by the broad $\mathrm{A}_{1 \mathrm{~g} .}{ }^{25}$ The broadening of the $\mathrm{A}_{1 \mathrm{~g}}$ mode reflects the complexity of the sample and is generally associated to partial inversion in mixed spinels where the same position is shared by more than one different cation. It is also interesting 
to remark that in the spectrum obtained at 3 hours, the otherwise very weak $\mathrm{T}_{2 \mathrm{~g}(2)}$ mode presents a discontinuity in the left branch at $457 \mathrm{~cm}^{-1}$, suggesting the higher cationic disorder of the manganese ferrite formed at this stage of the reaction with respect to the final product.

Considering all these factors we assume a three-step process to understand the morphological and chemical evolution of the magnetite CNCs after 8 hours of reaction (Scheme 1): (i) In the early stages of the reaction the nucleophilic attack of the acetate group over the cation center of $\mathrm{FeCl}_{3}$ under basic conditions leads to the formation of iron (III) hydroxide molecules in the reaction medium. The subsequent hydrolysis and condensation of such species produce the first oxohydroxide monomers that grow to form small lepidocrocite crystallites with concomitant feroxyhyte impurities. (ii) This metastable phase evolves to the more stable maghemite, while the coalescence of the crystallites takes place leading to the formation of the CNCs. In this sense, the interaction between the hydroxyl groups of EG and the surface of the iron oxide nanoparticles may help the clustering process. (iii) The maghemite CNCs evolve to magnetite thanks to the strong reducing power of PEG and EG at high temperatures. At the same time, the individual units that form the CNCs undergo digestive ripening and rearrange in a thermodynamically more stable disposition leading to larger grain sizes (as corroborated by TEM characterization and the thinner and more intense XRD peaks). As a result of this rearrangement the interstitial free space diminishes and the overall size of the CNCs is reduced.

Alternatively, the data presented herein highlights the complexity of the reaction mechanism that leads to the formation of $\mathrm{Mn}_{0.6} \mathrm{Fe}_{2.4} \mathrm{O}_{4} \mathrm{CNCs}$ (Scheme 1). Our findings suggest that in the early stages of the reaction a poorly crystalline Mn-doped lepidocrocite phase is formed. Interestingly, the phase transition in which lepidocrocite would evolve to form the spinel structure seems to be slowed down in comparison with the reaction in which magnetite CNCs are formed. As a consequence of the different kinetics, the $\mathrm{Mn}_{0.6} \mathrm{Fe}_{2.4} \mathrm{O}_{4} \mathrm{CNCs}$ are only formed after $3 \mathrm{~h}$ of reaction and the Ostwald ripening process previously reported for magnetite is strongly hampered for the time window of this experiment $(8 \mathrm{~h})$. These findings suggest that the doping of lepidocrocite with $\mathrm{Mn}^{2+}$ may improve the stability of this otherwise metastable phase.

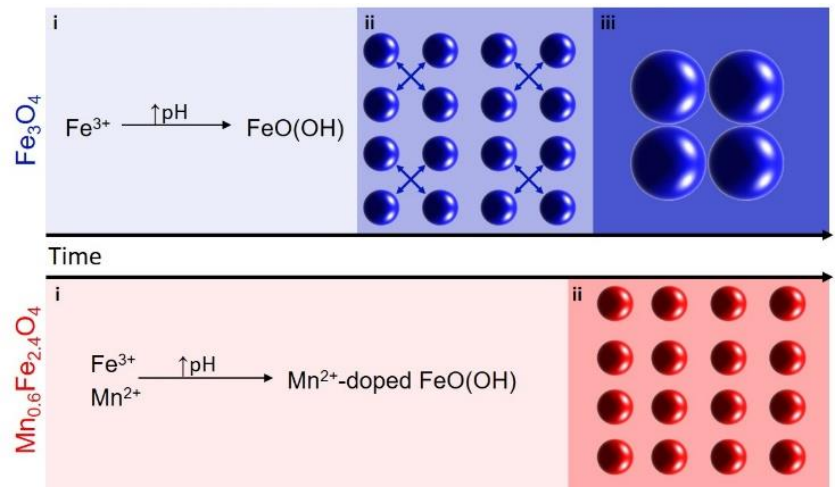

Scheme 1. Schematic representation of the synthetic mechanism towards the formation and growth of the units forming the CNCs.

Spinel ferrite CNCs may be particularly relevant for bio-related applications such as magnetic hyperthermia or MRI, where the magnetic coupling of the individual units of these secondary superstructures lead to interesting collective features. ${ }^{26}$ Therefore it is important to characterize the magnetic properties of the $\mathrm{Fe}_{3} \mathrm{O}_{4}$ and $\mathrm{Mn}_{0.6} \mathrm{Fe}_{2.4} \mathrm{O}_{4}$ CNCs obtained at the end of the synthetic protocol studied herein (Figure 4). Along these lines, both magnetization vs. magnetic field loops and magnetization vs. temperature (ZFC-FC) plots have been recorded. In terms of magnetization, both samples show high saturation $\left(\mathrm{M}_{\mathrm{S}}\right)$ values $\left(83.5 \mathrm{emu} / \mathrm{g}\right.$ for $\mathrm{Fe}_{3} \mathrm{O}_{4}$ and $96.2 \mathrm{emu} / \mathrm{g}$ for $\mathrm{Mn}_{0.6} \mathrm{Fe}_{2.4} \mathrm{O}_{4}$ ), being approximately $85 \%$ of their respective bulk values at $10 \mathrm{~K}$ (Figure $4 a, b)$. Even though there is a decrease in magnetization at room temperature due to thermal fluctuations $(73 \mathrm{emu} / \mathrm{g}$ for $\mathrm{Fe}_{3} \mathrm{O}_{4}$ and $71 \mathrm{emu} / \mathrm{g}$ for $\mathrm{Mn}_{0.6} \mathrm{Fe}_{2.4} \mathrm{O}_{4}$ ), the values reported are still very high, rendering these $\mathrm{CNCs}$ interesting materials for applications such as magnetic separation or hyperthermia, given their large total magnetic moment and diameter. These results are in opposition to previous studies where individual particles undergo an important decrease in saturation magnetization due to the formation of a superficial dead magnetic layer. ${ }^{27,28}$ Moreover, both samples present particularly high energy barriers, remaining in a blocked state throughout the entire temperature range used in this study (Figure 4c, d). Such interesting magnetic properties can be ascribed to the special confinement of the individual crystals into secondary superstructures, providing a strong magnetic coupling between neighbor units. Furthermore, the magnification of interfacial interactions may compensate the negative effect that spin disorder and other superficial phenomena typically have on the magnetic properties of this kind of nanocrystals. In the particular case of the $\mathrm{Mn}_{0.6} \mathrm{Fe}_{2.4} \mathrm{O}_{4}$ sample, the high saturation magnetization attained also stems from the specific stoichiometry of this material, with an increased $\mathrm{M}_{\mathrm{S}}$ stemming from the disposition of the different metallic cations and the way they are distributed in the spinel structure. $^{29}$
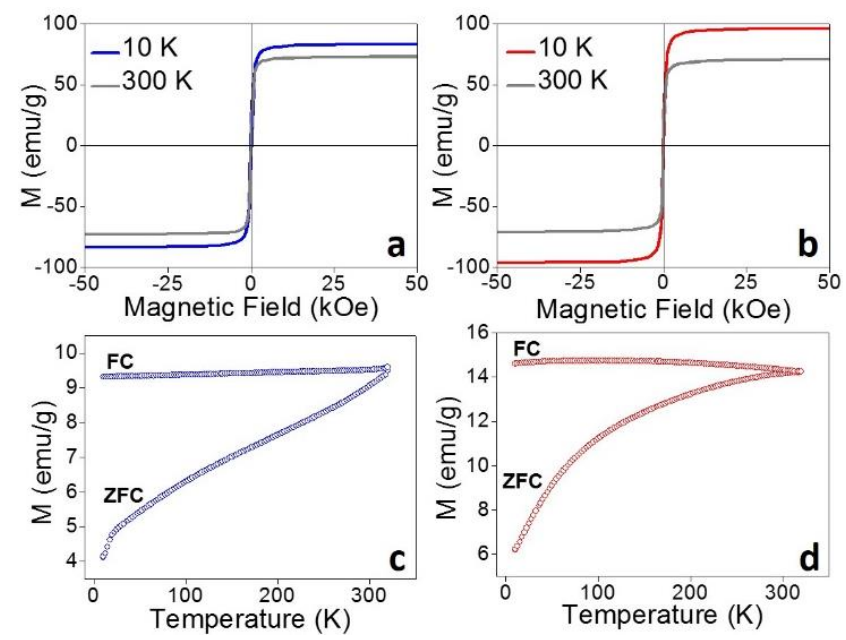

Figure 4. Hysteresis loops at different temperatures $(a, b)$ and temperature-dependent (ZFC-FC, $100 \mathrm{Oe}$ ) magnetization (c, d) of the $\mathrm{Fe}_{3} \mathrm{O}_{4}$ (blue) and of $\mathrm{Mn}_{0.6} \mathrm{Fe}_{2.4} \mathrm{O}_{4}$ (red) CNCs.

\section{CONCLUSIONS}

In conclusion, we herein present our study on the reaction mechanism behind the formation of $\mathrm{Fe}_{3} \mathrm{O}_{4}$ and $\mathrm{Mn}_{0.6} \mathrm{Fe}_{2.4} \mathrm{O}_{4}$ CNCs. We demonstrate that in both syntheses the final products evolve through a series of poorly crystalline intermediates. Firstly, a metastable lepidocrocite phase is obtained in the early stages of the reaction. Interestingly, the presence of interstitial $\mathrm{Mn}^{2+}$ cations in the structure of lepidocrocite affects strongly the stability of this intermediate and thus, has an important impact in the evolution of the magnetic structures to be attained. In a second step, the lepidocrocite phases evolve into a more 
stable spinel structure: maghemite (with subsequent reduction to magnetite) and non-stechiometric manganese ferrite, respectively. Furthermore the assembly of the nanocrystals formed into CNCs is only obtained once the spinel structure has been formed, following a rearrangement process in which the interstitial space between individual units is reduced along with the surface energy of the system. Depending on the relative stability of the lepidocrocite phase an Ostwald ripening process takes place, leading to an overall reduction in the size of the CNCs, with important consequences in the final magnetic behavior.

Alternatively, these results can be an example for further studies in which Raman spectroscopy (alone and/or in combination with other techniques) will be used as a powerful tool to study the poorly crystalline intermediates that are frequently obtained in the colloidal synthesis of nanoparticles. Such studies can indeed offer a better understanding of complex processes that remain nowadays elusive to the scientific community.

\section{ASSOCIATED CONTENT}

Supporting Information. TEM images, supplementary size histograms of the individual units and comparison of magnetite and Mn-ferrite XRD patterns. This material is available free of charge via the Internet at http://pubs.acs.org.

\section{AUTHOR INFORMATION}

\section{Corresponding Author}

* Email: miguel.comesana@uvigo.es

\section{Author Contributions}

The manuscript was written through contributions of all authors.

\section{Funding Sources}

The authors acknowledge financial support from the Xunta de Galicia (regional government, Spain) under the project ED 431C 2016-034 and from the Spanish Ministerio de Economía y Competitividad under project CTM2014-58481-R.

\section{Notes}

The authors declare no competing financial interest.

\section{REFERENCES}

(1) Xia, Y.; Xiong, Y.; Lim, B.; Skrabalak, S. E. Shape-Controlled Synthesis of Metal Nanocrystals: Simple Chemistry Meets Complex Physics? Angew. Chemie - Int. Ed. 2009, 48, 60-103.

(2) Xia, Y.; Xia, X.; Peng, H.-C. Shape-Controlled Synthesis of Colloidal Metal Nanocrystals: Thermodynamic versus Kinetic Products. J. Am. Chem. Soc. 2015, 137, 7947-7966.

(3) Thanh, N. T. K.; Maclean, N.; Mahiddine, S. Mechanisms of Nucleation and Growth of Nanoparticles in Solution. Chem. Rev. 2014, 114, 7610-7630.

(4) Mühlig, S.; Cunningham, A.; Dintinger, J.; Scharf, T.; Bürgi, T.; Lederer, F.; Rockstuhl, C. Self-Assembled Plasmonic Metamaterials. Nanophotonics 2013, 2, 211-240.

(5) Shiohara, A.; Wang, Y.; Liz-Marzán, L. M. Recent Approaches toward Creation of Hot Spots for SERS Detection. J. Photochem. Photobiol. C Photochem. Rev. 2o14, 21, 2-25.

(6) Khomskii, D. Classifying Multiferroics: Mechanisms and Effects. Physics (College. Park. Md). 2009, 2, 20.

(7) Otero-Lorenzo, R.; Fantechi, E.; Sangregorio, C.; Salgueiriño, V. Solvothermally Driven Mn Doping and Clustering of Iron Oxide Nanoparticles for Heat Delivery Applications. Chem. Eur. J. 2016, 22, 6666-6675. Materials by Design. Chem. Soc. Rev. 2o12, 41, 6874-6887. Kostopoulou, A.; Lappas, A. Colloidal Magnetic Nanocrystal Clusters: Variable Length-Scale Interaction Mechanisms, Synergetic Functionalities and Technological Advantages. Nanotechnol. Rev. 2015, 4, 595-624.

Grzelczak, M.; Vermant, J.; Furst, E. M.; Liz-Marzán, L. M. Directed Self-Assembly of Nanoparticles. ACS Nano 2010, 4, 3591-3605.

Jones, M. R.; Seeman, N. C.; Mirkin, C. A. Programmable Materials and the Nature of the DNA Bond. Science 2015, 347, 1260901.

Rikken, R. S. M.; Nolte, R. J. M.; Maan, J. C.; van Hest, J. C. M.; Wilson, D. A.; Christianen, P. C. M. Manipulation of Micro- and Nanostructure Motion with Magnetic Fields. Soft Matter 2014, 10, 1295-1308.

Schattling, P. S.; Ramos-Docampo, M. A.; Salgueiriño, V.; Städler, B. Double-Fueled Janus Swimmers with Magnetotactic Behavior. ACS Nano 2017, 11, 3973-3983.

Tan, R. P.; Carrey, J.; Respaud, M. Magnetic Hyperthermia Properties of Nanoparticles inside Lysosomes Using Kinetic Monte Carlo Simulations: Influence of Key Parameters and Dipolar Interactions, and Evidence for Strong Spatial Variation of Heating Power. Phys. Rev. B 2014, 9o, 214421.

Cannas, C.; Ardu, A.; Musinu, A.; Suber, L.; Ciasca, G.; Amenitsch, H.; Campi, G. Hierarchical Formation Mechanism of $\mathrm{CoFe}_{2} \mathrm{O}_{4}$ Mesoporous Assemblies. ACS Nano 2015, 9, 7277-7286.

Deng, H.; Li, X.; Peng, Q.; Wang, X.; Chen, J.; Li, Y. Monodisperse Magnetic Single-Crystal Ferrite Microspheres. Angew. Chemie - Int. Ed. 2005, 44, 2782-2785. Cheng, C.; Xu, F.; Gu, H. Facile Synthesis and Morphology Evolution of Magnetic Iron Oxide Nanoparticles in Different Polyol Processes. New J. Chem. 2011, 35, 1072-1079.

Scott, J. F. Soft-Mode Spectroscopy: Experimental Studies of Structural Phase Transitions. Rev. Mod. Phys. 1974, 46, 83128.

Kreisel, J.; Weber, M. C.; Dix, N.; Sánchez, F.; Thomas, P. A.; Fontcuberta, J. Probing Individual Layers in Functional Oxide Multilayers by Wavelength-Dependent Raman Scattering. Adv. Funct. Mater. 2012, 22, 5044-5049.

Liébana-Viñas, S.; Otero-Lorenzo, R.; Rivas-Murias, B.; Salgue. Concentric $\mathrm{MnFe}_{2} \mathrm{O}_{4}$-Rich core $/ \mathrm{Cr}_{2} \mathrm{O}_{3}$-Rich Shell Nanoparticles. J. Phys. Conf. Ser. 2014, 521, 12002.

Otero-Lorenzo, R.; Weber, M. C.; Thomas, P. A.; Kreisel, J.; Salgueiriño, V. Interplay of Chemical Structure and Magnetic Order Coupling at the Interface between $\mathrm{Cr}_{2} \mathrm{O}_{3}$ and $\mathrm{Fe}_{3} \mathrm{O}_{4}$ in Hybrid Nanocomposites. Phys. Chem. Chem. Phys. 2014, 16, 22337-22342.

Faria, D. L. A. De; Venâncio Silva, S.; de Oliveira, M. T. Raman Microspectroscopy of Some Iron Oxides and Oxyhydroxides. J. Raman Spectrosc. 1997, 28, 873-878.

Jubb, A. M.; Allen, H. C. Vibrational Spectroscopic Characterization of Hematite, Maghemite, and Magnetite Thin Films Produced by Vapor Deposition. ACS Appl. Mater. Interfaces 2010, 2, 2804-2812.

Shebanova, O. N.; Lazor, P. Raman Spectroscopic Study of Magnetite $\left(\mathrm{FeFe}_{2} \mathrm{O}_{4}\right)$ : A New Assignment for the Vibrational Spectrum. J. Solid State Chem. 20o3, 174, 424-430.

Pereira, C.; Pereira, A. M.; Fernandes, C.; Rocha, M.; Mendes, R.; Fernández-García, M. P.; Guedes, A.; Tavares, P. B.; Grenèche, J.-M.; Araújo, J. P.; et al. Superparamagnetic $\mathrm{MFe}_{2} \mathrm{O}_{4}(\mathrm{M}=\mathrm{Fe}, \mathrm{Co}, \mathrm{Mn})$ Nanoparticles: Tuning the Particle Size and Magnetic Properties through a Novel One-Step Coprecipitation Route. Chem. Mater. 2012, 24, 1496-1504. Lartigue, L.; Hugounenq, P.; Alloyeau, D.; Clarke, S. P.; Lévy, M.; Bacri, J.-C.; Bazzi, R.; Brougham, D. F.; Wilhelm, C.; Gazeau, F. Cooperative Organization in Iron Oxide MultiCore Nanoparticles Potentiates Their Efficiency as Heating Mediators and MRI Contrast Agents. ACS Nano 2012, 6, 10935-10949. 
(27) Berkowitz, A. E.; Schuele, W. J.; Flanders, P. J. Influence of Crystallite Size on the Magnetic Properties of Acicular $\gamma-$ $\mathrm{Fe}_{2} \mathrm{O}_{3}$ Particles. J. Appl. Phys. 1968, 39, 1261-1263.

(28) Chen, J. P.; Sorensen, C. M.; Klabunde, K. J.; Hadjipanayis, G. C.; Devlin, E.; Kostikas, A. Size-Dependent Magnetic Properties of $\mathrm{MnFe}_{2} \mathrm{O}_{4}$ Fine Particles Synthesized by
Coprecipitation. Phys. Rev. B 1996, 54, 9288-9296.

(29) Goodenough, J. B. Magnetism and the Chemical Bond; John Wiley And Sons, 1963.

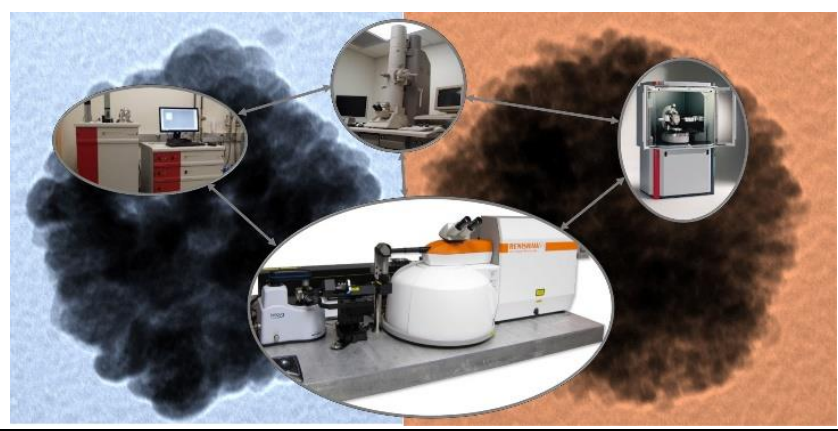

